\title{
Cirugía cardíaca correctora neonatal con circulación extracorpórea
}

\author{
Oscar García P. ${ }^{1}$; Oscar Gómez V. ${ }^{2}$; Claudjo Arretz V.:2 \\ Luis León M. '; Stephan Haecker D.'
}

\section{Neonatal cardiac surgery with cardiopulmonary bypass}

\begin{abstract}
A relrospecive review was done of ninereen newborn intonts with rotal anomalcus pulmonary venous drainage and twenty one neonates with transposition of the great arleries, all operated under cardiopulmonary byposs at the neonatal period, from january 1990 throughout june 1992, to describe results of surgery, complicalions, lelhatity and prevention of these laje events. Total anomabus pulmonary venous drainage wos supracardiac in ten cases. inlracadioc in three, and infracardiac in six inlanis. Diagnosis wos confimed by echocardiography. All linese potienis werc operaled in funclional capocily $N$ at an average age of 16 days. Two patienls died of surgery and one infant later. Pulmonary venous obstsuction was delecled at follow up in three polients, one of them has been already operaled for thal. In neonales with transposition of the great arteries, average age al operation was 10,5 days, in eighteen coses diggnosis was done by echocardiogrophy and cordiac cakhoterism; ail were operated in lunctional capacily IV and four under treatmenl with proslaglandin intravenous infussion. Arlerial swilch (latene's operotion) wos done in eighteen coses and alrial switch 'Senning's operotiont in the remaining three infonts. Two polients of those lreated with the jalene' procedure died al surgery. Three infonts hod stenosis of the pulmonory allery af follow up, whict was monoged by balloon dilatation in two of them, and one patient had pulmonary venous obstruction after an atrial switch. which has been alseady operated. One infant hod supraventricular paroxiștic lachycardia, and was managed with amiodarone. In order to reduce surgical morlalily, early diagnosis, inmediate transpert to a cordiovoscuiar center, use of proslaglandin introvenous infussion in polienls with corigenila! heart diseoses which are dependent on duclus patency. and improvement of functional capacity before surgery with aggressive medical treotment orc mandatory for these parierls.
\end{abstract}

Key words: Corrective cordiac surgery, cardiopulmonary bypass, neonoles, prevention morlalily.)

Las malformaciones cardíacas congénitas (MCC) ocurren aproximadamente en $1 \%$ de los recién nacidos vivos. Si se dejan a su evolución natural, constituyen una de las principales causas de mortalidad infantil, $70 \%$ fallecen antes del ano te vida y $33 \%$ en el primer mes ${ }^{1-3}$.

Se conocen más de cuarenta malformaciones cardiacas congénitas distintas, siendo las más frecuentes los defectos septales interventricular (DSIV) e intcrauricular (DSIA), ductus arterioso persistente, estenosis pulmonar, tetralogía de Fallot, coartación aórtica, estenosis aórtica, uansposición de grandes arterias (TGA), drenaje venoso anómalo pulmonar total (DVAPT), atresia

1. Centro Candiovascular, Hospital Luis Calvo Mackenกa. tricúspidea, canal auriculoventricular y ventriculo único ${ }^{1-3}$.

Algunas de estas malformaciones pueden ser corregidas precozmente en la vida, mediante intervenciones quinírgicas sin circulación extracorpórea, como coartación de la aorta, intersupción del cayado aóttico, estenosis aórtica y ductus arterioso persistente, pero otras, como la TGA y el DVAPT requicren de dicho procedimiento de derivación curculatoria. Para describir el mancjo perioperatorio de estas últimas, considerando la técnica quirúrgica empleada; su mortalidad hospitalaria, las causas de esta y las complicaciones postoperatorias, se revisaron todos los casos de recién nacidos operados por transposición de grandes arterias y drenaje venoso anómalo pulmonar total, entre enero de 1990 y junio de 1992. en el Centro Cardiovascular del Hospital Luis Calvo Mackenna de Santiago. 


\section{Material y Método}

Se revisaron retrospectivamente las historias clínicas de los recién nacidos operados de TGA y DVAPT durante el período indicado, registrando edad y peso al momento de la operación, procedencia, tnétodo utilizado para el diagnóslico, operación efectuada, monalidad hospitalarja y sus causas, seguimiento y complicaciones.

Se consideró que la causa de muerte era de origen car díaco cuando ésta se asoció a choque cardiogénico, insu ficiencia cardíaca refractaria a tralamienlo, o mala contractilidad miocárdica al término de la circulación extracorpórea: y debido a falla orgánica múliple si habia alteración furcional de tres o más sistemas. La capacidad funcionaI (CF) de los pacientes se califić de acuerdo a la clasificación de "New York Heart Association".

\section{Resultados}

En el periodo de estudio se operaron 19 recién nacidos con DVAPT y 21 con TGA.

La edad al momento de la operación de los niffos con DVAPT fluctuaba entre 2 y 28 días, media de 16 dias; el peso entre 2600 y 4070 , media 3171 y $13(68,5 \%)$ casos venían de regiones ajenas a la metropolitana de Santiago. El diagnóstico en todos ellos se efectuó por ccocardiograma, en diez casos el drenaje venoso era supracardíaco, en tres cardíaco y en seis infracardíaco y todos se operaron en CF IV. Tres recién nacidos operados por DVAPT fallecieron, dos tenían DVAPT supracardíaco (uno murió en la operación por causa cardíaca y otros tres meses después de operarse, por falla orgánica múltiple); el tercer paciente, portador de DVAP intracardíaco, murió en el postoperatorio reciente. El seguimiento se ha efectuado en los sobreviventes por entre 3 y 27 meses, promedio 13,5 meses. Tres niños sufrieron obstrucción de las venas pulmonares, uno a los dos meses de operado y falleció en el hospital de origen; dos a los 4 meses de operados (uno ya fuc corregido quirúrgicamente, el otro espera operación).

La edad al momento de la operación de los niños con TGA fluctuaba entre 3 y 28 días, promedio 10,5 dias. Los pesos fluctuaban entre 2600 y $4060 \mathrm{~g}$, promedio $3433 \mathrm{~g}$. Trece pacientes $(62 \%)$ provenian de otras regiones del país, el resto de Santiago. En tres el diagnóstico se efectuó sólo por ecocardiograma, en los otros $18 \mathrm{se}$ empleó, además, cateterismo cardiaco, con el objeto de realizar septostomía de Rashkind. En 18 casos la TGA no se acompañaba de DSIV, la que estaba presente en los otros tres. Todos se operaron en CF IV, en cuatro se usó prostaglandina El en infusión intravenosa para mantener permeable el ductus arterioso. Se efectuó reimplantación ("switch") arterial (operación de Jatene), en 18 (15 sin DSIV), y en tres reimplantación de las auriculas (operación de Senning). La indicación del procedimicnto de Senning en estos nifios se hizo por ventrículo izquierdo de tamaño pequeño en uno y alteraciones anatómicas de las arterias coronarias en los otros dos. Estas últimas consistian, en un caso, en coronaria derecha dominante e izquierda intramural y, en el otro, origen de la arteria circunfleja en la coronaria derecha. La letalidad estuvo dada por dos niños operados con técnica de Jatene, que fallecieron en el intraoperatorio por causa cardíaca. El seguimiento en los 19 sobrevivientes se ha extendido entre 2 meses y 2 años 5 meses, promedio 17,2 meses, mostrando que un recién nacido operado con procedimiento de Senning presento obstrucción de venas pulmonares a la edad de 8 meses, de lo que ya fue operado. Entre los niños operados con técnica de Jatene, uno presento estenosis supravalvular pulmonar y otro estenosis en el origen de ambas ramas pulmonares, que en ambos casos fueron traladas con dilatación por medio de balones en caterismo cardíaco, mientras un tercero tiene estenosis en el origen de la arteria pulmonar izquierda y está esperando para dilatación con catćter. Otro niño ha cursado con taquicardias paroxisticas supraventriculares y está siendo manejado con amiodarona.

\section{Comentario}

Las malformaciones cardíacas congénitas cianóticas son las de presentación clínica más grave y precoz en la vida, requiriendo, en general, cirugía correctora o paliativa pronta. La letalidad quirúrgica varía en distintos centros y depende del tipo de malformación, del estado hemodinámico del paciente a la operación, la oportunidad del diagnóstico y la intervención, el peso y la edad gestacional al nacer, la presencia de lesiones cardíacas asociadas y la magnitud de éstas. Es así como se describe mortalidades hospitalarias entre 11 y $36 \%$ para el caso de los DVAPT operados en recién nacidos ${ }^{4-6}$, de 5 a $11 \%$ para operaciones de Senning y de 12 a $15 \%$ para procedimicntos de Jatene ${ }^{7-12}$ en pacientes con 
TGA, siendo similares nuestros resultados en ambos casos. En 10\% de los sobrevivientes de DVAPT operados se registra obstrucción del drenaje venoso4.

La gran cantidad de pacientes derivados de provincia en esta serie, indudablemente influyó en la edad algo tardía en que llegaron a operarse, considerando que deben ser trasladados al centro tcreiario y sometidos a procedimientos de diagnóstico antes que a cirugía.

La principal causa de muerte fue cardíaca, to que habla más que rada de la gravedad del estado en que Ilegan estos neonatos al quirófano, debido a la complejidad de su afección cardíaca, en contraste con el escaso número de ellos que fueron tratados con prostaglandina, para mantener el ductus permeable y aumentar el flujo sanguínco pulmonar.

Las técnicas quirúrgicas y el manejo postoperatorio han evolucionado positivamente con el liempo, lo que permite ser optimista en cl sentido de que será posible, en el futuro, disminuir cada vez más la mortalidad de los recién nacidos con malformaciones cardíacas graves, a lo que debe contribuir la pesquisa y el traslado cada vez más precoces de estos niños a los centros quirúrgico cardiovasculares habilitados para resolver su problema, así como el empleo más generalizado de infusiones de prostaglandina en los casos de malformaciones en que la perfusion pulmonar y la vida dependen de la persistencia de la permeabilidad del ductus arterioso, como algonas formas de TGA, tetralogía de Fallot extrema, atresia pulmonar con septum intacto e interrupción del cayado ártico ${ }^{13},{ }^{14}$, pues, de este modo será posible mantener al recién nacido en las mejores condiciones hemodinámicas, permitiéndosele enĺrentar la cirugía con más posibilidades de sobrevida.

\section{Resumen}

Se revisaron retrospectivamente las fichas clínicas de recién nacidos operados con circulación extracorporea por drenaje venoso anomalo pulmonar total (19 nifos) y transposición de grandes arterias (21 pacientes) entre los meses de enero 1990 y junio 1992 . En 10 nifos el drenaje venoso anómalo era supracardíaco, en tres cardíaco y en seis infracardíaco; el diagnostico correcto fue hecho en todos por ultraso- nografía; la edad promedio a la operación eta 16 días; todos los pacientes con drenajes venosos anómalos fueron operados en capacidad funcional IV; dos niños fallecieron en el hospital y uno tadíamente, después de su egreso; en cl seguimiento de los sobrevivientes se registró obstrucción de las venas pulmonares en tres casos, uno de los cuales ya fue reoperado. Entre los neonatos operados por transposición de grandes arterias, el dianóstico correcto fue hecho en 18 mediante ecocardiografía y cateterismo cardíaco, en los olros tres sólo por el primer procedimicnto; todos se operan cn capacidad funcional IV, cuatro recibieron infusiones intravenosas con prosta. glandina E1; la edad promedio a la operación era 10,5 días. Se efectuó operación de Jatene o reimplantación arterial en 18 y de Senning o reim. plantación auricular en tres; la letalidad hospitalaria estuyo dada por dos casos sometidos a reimplantación arterial. En el seguimiento, tres niños operados por transposición arterial mostraron cvidencia de estenosis de arterias pulmonares (dos fueron tratados con dilacioncs mediante catéteres provistos de balones, el otro espera igual proceder) y uno obstruccion de venas pulmonares (Senning), que ya ha sido reoperado; otro más sufre de taquicardias paroxísticas supraventriculares y está en tratamiento médico. Para mejorar la mortalidad, el diagnóstico y el trasiado a un centro de atención terciario deben ser precoces, es preciso generalizar uso de prostaglandina en cardiopatías congénitas en que la perfusión pulmonar y la supervivencia dependen de la permeabilidad del ductus arterioso y mejorar el estado hemodinámico de los pacientes antes de operarlos, con tratamiento médico agresivo.

(Palabras clave: cirugía cardiaca correctora, neonatos, circulación extracorpórea, prevención mortalidad).

\section{Referencias}

1. Eimbcke F, León L: Cardiopatías congénitas en el recién nacido. En: Artaza O, Zillenuelo R. Manual de Cardiología y Cirugía Cardiovascular Infantil, Santiago. Editorial Mediterráneo, 1989: 38-45.

2. Fyler D, Trends. En: Fyler D: Nadas ${ }^{+}$Pediatric Cardiology, Philadelphia: Edil. Hanley and Belfus, INC, 1992; 273-280.

3. Boniels S.: Epidemiotogy. En: Long W. Fetal and Neonatal Cardiology, Philadelphin: Edit. W.B. Saunders Company, 1990; 425-436. 
4. Fyler $D$. Total anomalous pulmonary venous returtss. En: Fyler D. Nadas' Pediatric Cardiology, Philadelphia: Hanley and Belfus RVC.. 1992; 683-691.

5. Russell $L$, Konberly $K$ : Anomalous venous connections, pulmonary and sistemic. En: Adams F, Emmanoulides $G$, Riernenschneider T. Heart Disease in Infants, Children and Adolescents, Baltimore: Wilkins and Wilkins Co. 1989; 597 .

6. Galloway A, Campbell D, Clarke D: The value of early repair for total anornalous pulromary venous drainage. Pediatr Cardiol 1985: 6: 77,

7. Fyler $D:$ D-Transposition of the great arteries. Fn: Fyler D. Nadas' Pediatric Cardjology, Philadelphia: Haniey and Belfus LNC., 1992; \$57-573.

8. Milton $P$ : Complete Iransposition of the great arteries. Es: Adams F, Emmanoulides G, Ricmenschneider T. Heart Discase in Infants, Children and Adolescents, Baltumore: Wilkins and Wilkins Co., 1989: $371-417$.

9. Trusler $G$, Castañeda A, Rarenthal A et al.: Curtent results of management in transposition of the great ancries, with special emphasis on patients with associated ventricular seplal defect. J Am Coll Cardjol 1987; 10: 1061 .

10. Quaegebew $J$, Rohmer $J$, Ottenkamp $f$ es al, The arterial switch operation. An eigth-yeat experience. I Thorac Cardiovasc Surg 1986; 92: 361-384.

11. Brawn $W$, Mee $R$ : Early results for anatomic correction of transposition of the great arteries and for doubleoutlet right ventricle with subpulmonary ventricular septal defect. J Thorac Cardiovasc Surg 1988; 95: 230-238.

12. Idriss $F$, Jibawi $M, D_{e}$ Leon $S$ et al : Anerial switch in simple and connplex transposition of the great arreries. J Thorac Cardiovasc Surg 1988; 95: 29.

13. Tainer $N$ : Heart falure. En: Adams F, Emmanouljdes $G$, Riemenschneider $T$. Heart Disease in Infants, Children and Adolescents, Baltimore: Wilkins and Wilkins Co., 1989; 908.

14. Heymon $M$ : Phatmacologic use of prostaglandin $E_{1}$ in infants with congenilal heart disease. Am Heart J 1981; $101: 837-843$. 